\title{
CD44 Positive
}

National Cancer Institute

\section{Source}

National Cancer Institute. CD44 Positive. NCI Thesaurus. Code C147057.

An indication that CD44 expression has been detected in a sample. 